\title{
Know Your Customer (KYC) based authentication method for financial services through the internet
}

\author{
Prakash Chandra Mondal ${ }^{1, *}$ \\ ${ }^{1}$ Department of Computer Science and Engineering, \\ United International University, \\ Dhaka, Bangladesh, \\ *prakash.cse2009@gmail.com
}

\author{
Rupam Deb ${ }^{2}$, Mohammad Nurul Huda ${ }^{3}$ \\ ${ }^{2}$ School of Information and Communication Technology, \\ Griffith University, \\ Brisbane, Australia, \\ rupam.deb@griffithuni.edu.au \\ ${ }^{3}$ Department of Computer Science and Engineering, \\ Dhaka, Bangladesh, \\ mnh@cse.uiu.ac.bd
}

\begin{abstract}
Financial services through the internet are running under various threats like phishing, pharming (cyber attack intended to redirect a website's traffic to another fake site), malware, and evolving sophistication of compromise techniques. Multi-factor authentication (MFA) financial service system alleviates the risk and makes it secure. Various methods of MFA run in troubles like the authentication device lost or stolen, false sense of security (if used on login device), compromised answer of the generic question, higher implementation costs, unambiguous movement profile, calculated hash value stolen (there is no chance to replace it), etc. Compliance with Anti-Money Laundering (AML), Know Your Customer (KYC) and sanction requirements continue to be a key focus area for Financial Institutions' (FIs) management; firms must ensure that they are following appropriate compliance procedures to meet the increasing regulatory demands [1,2]. By addressing existing limitation of MFA, this paper proposes dynamic KYC based MFA authentication method to ensure the secured access of the financial services through the internet. Analysis and simulation results show that the proposed method provides control same as existing MFA/2FA techniques, but customers will get relief from holding additional security devices that incur huge cost.
\end{abstract}

Keywords-multi-factor authentication (MFA); Know Your Customer (KYC); online banking authentication; internet banking; strong authentication; web security;

\section{INTRODUCTION}

Internet based financial services like balance transfer, ecommerce transaction, bill payment, investment to bank products (saving certificate, fixed deposit revenue) etc. are the appealing ways of doing business as well as performing all financial transaction location independently. As a result of these overwhelming facilities, it poses the highest point of risk as it uses public network over the world. The main endeavor of the banks and non bank financial institutions are to provide a consistent, secured and high available process of authentication to their customers with minimizing potential avenues of attack, especially attacking vectors beyond the control of either the customers or the Financial Institutions (FIs).

However, authentication methods for internet based financial system are still pursuing to ensure the highest level of security. Existing strong authentication methods sometimes make its nuisance to the genuine users who are operating their regular business using internet based financial system. Current activity of any of the users has a linear relationship with user's many past usage patterns such as nature of Uniform Resource Locator (URL) navigation, access geo-location, regular transaction nature, regular transaction range, regular transaction timing, transaction performing hardware address, natural transaction frequency, regular transaction purpose, biometric behavior, and many other non bio-metric behaviors.

In this research paper, Know Your Customer (KYC) information verification technique has been introduced as Challenge Question (CQ) during login using user ID and Password in order to verify user more intensively. In that case KYC must be privatized with widespread dynamic user input. The KYC database enriches from account opening initial data, user interaction and dynamic update through the application; on the other hand user can add more confidential information or random question/questions with answer/answers to the KYC database to make the authentication process much stronger and secured. Ranking on the KYC information will also be considered to be used as CQ; CQ will be asked to the user during login after success in user ID and Password verification. One or more CQ will be assigned to ask the user based on the risk factors assessment result. Top ranked CQ will be asked to the user when the risk assessment result is comparatively higher; on the other hand low ranked CQ will be asked for lower risk.

The rest of the paper is organized as follows. Section II overviews the related work. Description of our proposed model is introduced in Section III. Section IV discusses the result and compares with other methods of MFA. Finally, Section V concludes the paper with fruitful remarks.

\section{RELATED WORK}

In recent couple of decades, many authentication methods have evolved. A variety of technologies and methodologies have been exercised by the financial institutions in order to authenticate customers. These methods in the financial institutions include the use of customer passwords, personal identification numbers (PINs), digital certificates using a public key infrastructure (PKI), physical devices such as smart cards, one-time passwords (OTPs) [12], graphic-based authentication techniques[9], two-party authentication protocol [6], USB plug-ins or other types of "tokens", transaction profile scripts, biometric identification, and others. The level of risk protection afforded by each of these methods 
varies with the importance given by an individual institution. The selection and use of authentication technologies and methods should depend upon the results of the financial institutions' risk assessment processes [3, 5, 6, 8, 10]. Multifactor authentication (MFA) methods used by the banks evaluate the types of security mechanism adopted and develop security measures to reliably authenticate customers remotely accessing internet-based financial services; the study [4] by O.B. Lawal, A. Ibitola, O.B. Longe, 2013 addressed conducting risk-based assessments and customer awareness program. Providing foolproof security for financial applications is a rigorous activity. Security architect needs to consider various design considerations to make the applications bullet proof. White paper of Infosys limited by S. K Shivakumar and B. Krishnamurthy [5] present Advanced Security Design for financial applications' adaptive authentication (risk evaluation and multi factor authentication), which considers two factors: Digital Certificate Security and One Time Password (OPT) support. S. F. Shazmeen and S. Prasad [7] proposed challenge/response-based short-time password authentication method using symmetric cryptography in combination with a Software Security Model. The short-time password solution is used in software-based security systems; the encoding and decoding of information are done in specialized security software.

Challenge/response based Internet banking considers some authentication techniques, where one based on short-time password, one certificate-based, and other relates them to the taxonomy. We further outline how these solutions can be easily extended for non-repudiation (i.e., transaction signing) and for more sophisticated content manipulation attacks.

Some of the factors considered by the Business Signatures solution are [12]:

Credential Risk: Does the user have the correct password and other personal data expected?

Transaction Risk: Is the user trying to make a payment over a threshold amount or change passwords or other personal security information?

Location Risk: Is the user coming from an approved or previously authenticated Internet location with an expected Browser Profile and Computer Profile?

Behavioral Risk: Is the user coming at an unusual time of day, performing a transaction involving an unusual payee, or unusual amount given those users’ past behavior?

\section{PRoposed MODEL}

In this model of authentication for financial application, some techniques of comprehensive risk factor judgment have been proposed to identify a suspicious login over many existing risk evaluation by analysis of user's historical activities data, and also proposed a new authentication method based on KYC information for authorizing the user during login as well as before performing transaction. In this method CQs are choosing from a collection of CQs where the level of the CQ is defined by the risk factors calculation result. Risk factors are calculated considering Credential Risk (New user, Failed login attempt, User with no/very few identity information, Changing nature of transaction), Behavioral Risk
(Changed general transaction timing, Exceeded regular transaction frequency, Changed regular transaction purpose, Called to a new URL, which did not call by this particular user previously), Transaction Risk (Exceeded regular transaction limit, Exceeded profile transaction limit), and Location Risk (Changed geo-location, Changed transaction device). For appropriate and secure transaction using application, proposed model performs two operations. First one is risk factor calculation and second one is assigning the CQ based on the risk assessment result. The brief idea of the model is depicted in the Fig. 1. In the figure the initial step is login of the user with user ID and Password verification like other online applications. The forwarding stage is risk analysis for the login succeeded user. Next stage assign one or more CQ based on risk level formed by the result of prior stage. Final stage of the verification is OTP / EMAIL / OTP \& Email confirmation if it is indicated by the result of risk analysis. In some other cases confirmation stage may not be applicable where CQ is in final stage before performing and committing a transaction. The CQ based authentication applies immediately after login and before performing and committing a transaction to verify the user rigorously. This CQ replaces the 2FA or traditional question and answers mechanism from some other existing authentication models.

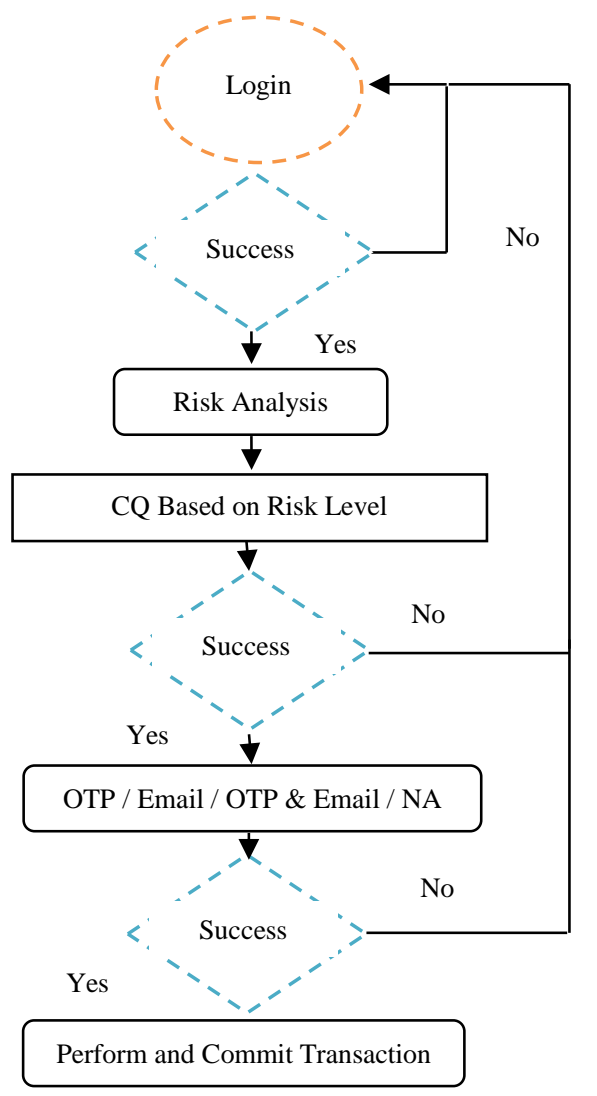

Fig. 1. Authentication model using CQ from the KYC information database

\section{A. Risk Factor Calculation}

Risk and threat in online transaction depend on various factors. The prime goal of the FIs is to permit right user for accessing financial network and restricting all others. In this context, it is necessary to analyze previous activities of the 
user and calculate similarity between previous and present by introducing multiple techniques and algorithms.

1) Behavioral Risk

Behavior-based model defense is based on the analysis of the exploit's interaction with the target. Interactions outside the normal behavior groups would be suspicious and quarantined. This method then has the potential to detect and analyze potential zero-day exploits in real time (Alosefer \& Rana, 2011) [11].

In this paper we have used hidden Markov model (HMM) for transitional probability of user's behavioral risk predictions (different than users general transaction timing, exceeding regular transaction frequency, different from regular transaction purpose, different from regular URL navigation etc).

a) User access time similarity assessment from general transaction timing

User behavior is assessed in this scope by computing the probability of the current access time from the previous access time log data. Activities of the user in the financial application can be collected from the comprehensive user log of the software which has been introduced behind the financial application to record different kinds of user activities during his/her navigation over the financial application. For this simulation live activities log from a client web based application [14] is used where hourly access time for user-I and user-II are depicted in Fig. 2 and Fig. 3, respectively. Time dependency of the user access has been partitioned hourly basis for each hour in 24 hours using equation (1).

Number of access of a particular user is $C_{h}$, where

$$
\mathrm{C}_{h}=\sum_{t=h-1}^{t=h+1} a^{t \pm \nabla t}
$$

Where $h$ represents current hour

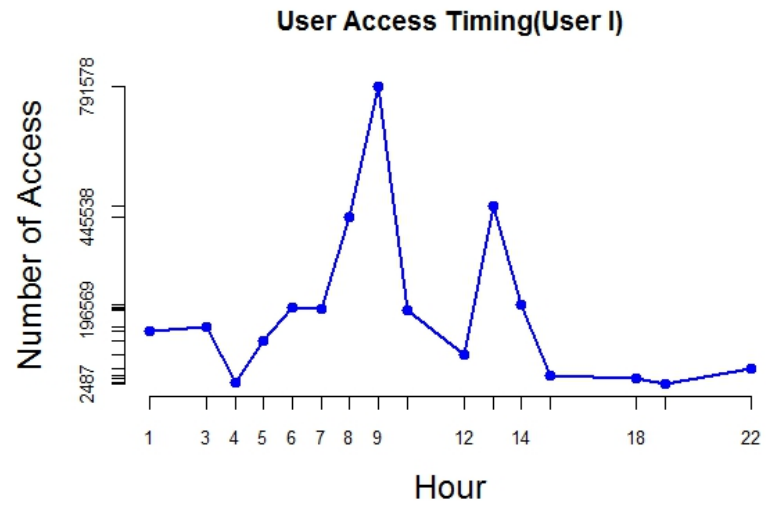

Fig. 2. Historical access time for user-I plotted by R studio

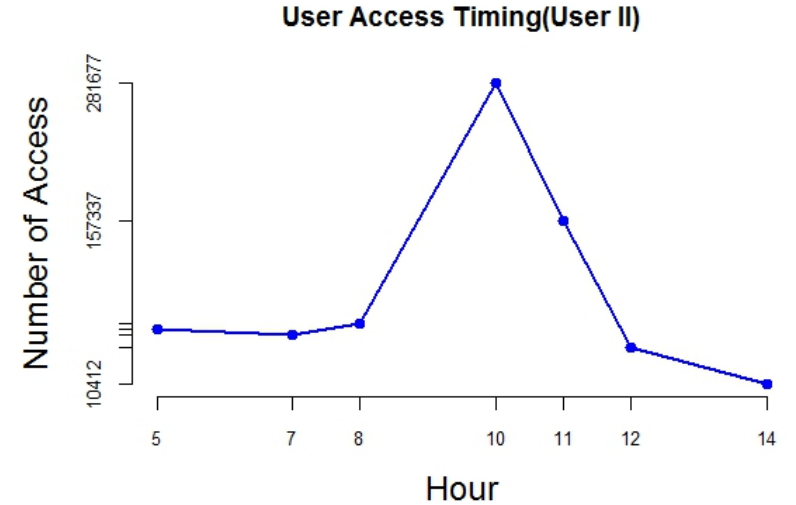

Fig. 3. Historical access time for user-II plotted by R studio

From the above behavioral access time data, the probability of using the application in the current time can be determined. The probability result determines the selection of CQ which will be assigned for the user on the login time. The probability of the current time from the historical timing of a particular user is calculated using HMM (2).

$\mathrm{P}(\mathrm{x}, \pi)=\mathrm{P}(\mathrm{x} 1, \ldots, \mathrm{xN}, \pi 1, \ldots . ., \pi \mathrm{N})=\mathrm{P}(\mathrm{xN} \mid \pi \mathrm{N}) \mathrm{P}(\pi \mathrm{N} \mid$ $\pi \mathrm{N}-1) \ldots . . \mathrm{P}(\mathrm{x} 2 \mid \pi 2) \mathrm{P}(\pi 2 \mid \pi 1) \mathrm{P}(\mathrm{x} 1 \mid \pi 1) \mathrm{P}(\pi 1)=\mathrm{P}(\pi 1) \mathrm{P}(\mathrm{x} 1$ $\mid \pi 1) \prod_{N=2}^{N} P\left(X_{N} \mid \pi_{N}\right) P\left(\pi_{N} \mid \pi_{N-1}\right)$

CQ level will be assigned based on the derived probability result. Less probability indicates suspicious or fake user; on the other hand high probability means trusted and real user. Suspicious user is checked by highly ranked CQ and vice versa.

b) Assessment of least time intervals between transactions

In this assessment process least time difference between two consecutive transactions from a dataset [14] of a live website has been measured. According to our model this assessment result can be applied after performing one or more transaction and before beginning the next transaction. User activities time records are obtained from the user activities log shown in Fig. 4 and assessed over 10 least intervals from the collection, which is figured out in the Fig. 5 by line chart. Fraudsters always try to commit transaction within a short time or using some automated script. Using this evaluation it has been considered short time intervals between the transactions which will prevent running script and quick transactions. On the other consideration we assessed users' regular short time intervals between the transactions which will match with users' regular behavior of transaction to indentify real user. 
Time Interval Between Transaction

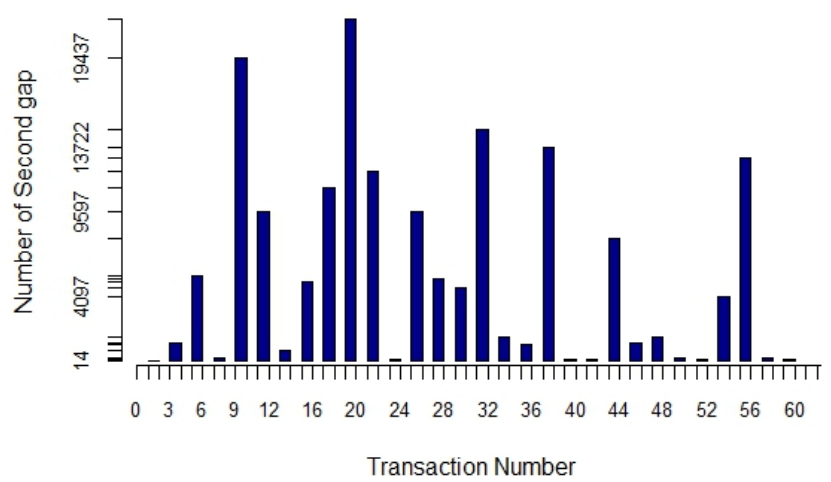

Fig. 4. Time interval between the transactions plotted by R Studio

\section{Least Gap Transaction}

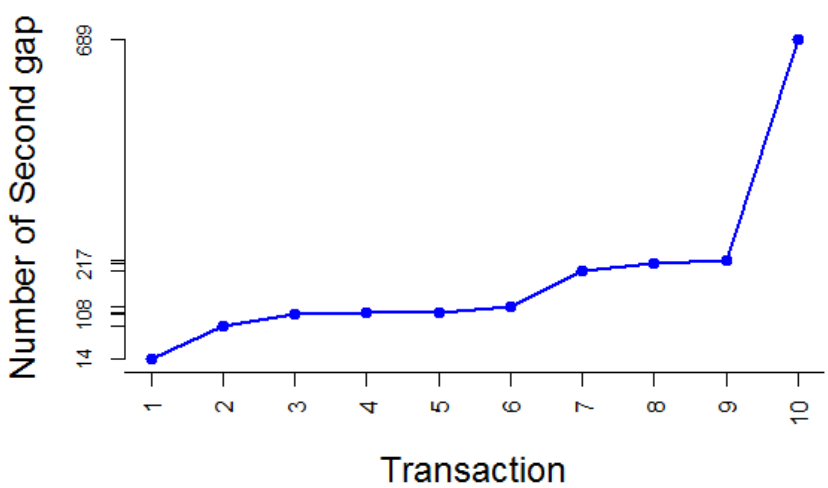

Fig. 5. Least time interval among consecutive quickest transactions plotted by R Studio

Any unnatural time gap between the transactions will be treated as high risk transaction. If two transactions are performed quickly within small time gap (30 seconds or less based on the application response time), it will temporarily block the transaction and notify the user as it may be generated by fraudster. In other case, if any transaction occurs in less than minimum time interval of users' previous activities will result to face a high rated CQ during transaction.

\section{c) Assessment of users URL traverse}

In this assessment all the navigation will be recorded and compared with previous traversal history. The proposed model will find out difference between the navigation attitudes. In case of new attitude the CQ selection process will carry a new weight to select hard (high ranked) CQ during login. This assessment is only applicable in the portal where number of URL and pages is very high and user requested to login into an unusual URL which is different from user's historical behavior. Sample of the traversal history log from live website [14] has been depicted in the Fig. 6.

\begin{tabular}{lll}
\hline uid & URL & DateTime \\
32 & /actiontracker/ & $2016-06-24$ 22:36:08 \\
32 & /actiontracker/login.php & $2016-06-24$ 22:36:09 \\
32 & /actiontracker/login.php & $2016-06-24$ 22:36:32 \\
32 & /actiontracker/tm_update.php & $2016-06-24$ 22:36:33 \\
32 & /actiontracker/tm_changedeadline_dtl.php?ta: 2016-06-24 22:36:34 \\
32 & /actiontracker/tm_changedeadline_dtl.php?ta: 2016-06-24 22:36:34 \\
32 & /actiontracker/tm_changedeadline_dtl.php?ta: 2016-06-24 22:36:34 \\
32 & /actiontracker/tm_changedeadline_dtl.php?ta: 2016-06-24 22:36:35 \\
32 & /actiontracker/th_update_to_tm.php & $2016-06-24$ 22:37:26 \\
32 & /actiontracker/th_update_to_tm_dtl.php?gid=: 2016-06-24 22:37:27 \\
32 & /actiontracker/ & $2016-06-2502: 30: 22$ \\
32 & /actiontracker/login.php & $2016-06-2502: 30: 23$ \\
32 & /actiontracker/ & $2016-06-2502: 35: 21$ \\
32 & /actiontracker/login.php & $2016-06-2502: 35: 21$ \\
32 & /actiontracker/login.php & $2016-06-2506: 02: 17$ \\
32 & /actiontracker/login.php & $2016-06-2506: 02: 17$ \\
32 & /actiontracker/tm_update.php & $2016-06-2506: 02: 18$ \\
32 & /actiontracker/tm_changedeadline_dtl.php?ta: 2016-06-25 06:02:19 \\
32 & /actiontracker/tm_changedeadline_dtl.php?ta: 2016-06-25 06:02:19 \\
32 & /actiontracker/tm_changedeadline_dtl.php?ta: 2016-06-25 06:02:19
\end{tabular}

Fig. 6. Historical log of user navigation taken from Nevicate for MYSQL

\section{2) Credential Risk}

In case of credential risk, this paper proposes a strong authentication method as all kinds of credential risk are vital and seldom used i.e personal information changing, failure to provide correct details. These criteria make a match between provided input and KYC database existing data. If user is failed to provide correct answer, the KYC information will be treated as suspicious and high ranked CQ will be assigned for further verification.

\section{3) Transactional Risk}

Transactional risk assessments are based on the KYC profile data limit and regular transaction domain. When user exceeds maximum value in respect of regular transaction or is short of minimum value of regular transaction, then it will be treated as suspicious transaction and high ranked CQ will be applied during the transaction. In case of exceeding the transaction profile (TP) upper limit by the user, the transaction will block the account temporarily without asking for CQ and any other MFA. From the World Bank dataset [13] to detect suspicious transaction standard deviation can be calculated as $\mathrm{STD}=\sqrt{\frac{\sum(X-\bar{X})^{2}}{n}}$, maximum and minimum of the last 30 to 100 transactional data.

Hence, MAX+STD $<$ Suspicious transaction amount $<$ MINSTD

\section{4) Location Risk}

Location risk is determined by translating the IP address to location. User will be asked on different occasions to keep record for his/her upcoming movement into the system. The system will compare the given information and current location prior to giving access to the system or making a transaction. In case of hardware, address used by the user will be compared with the current hardware (desktop, laptop, mobile, tab, etc.). If the hardware used for the transaction is different from all previous hardware/devices, the CQ will be harder based on the dissimilarity. 


\section{B. Assigning the CQ from the risk analysis}

More personalized information will be high ranking and more public questions will be rated as low value. Based on the risk factor taxonomy the challenge question will be transmitted to the user for further verification during login or prior to the transaction. The TABLE I. describes some of the samples of ranking method of the Challenge Question (CQ). Frequently asked question (FAQ) for the user will be pop-up to the user during his/her work through the application and the answer will be updated accordingly.

TABLE I. SAMPLE CHALLENGE QUESTION (CQ) WITH GRADING

\begin{tabular}{|c|c|c|}
\hline $\begin{array}{l}C Q \\
\text { No. }\end{array}$ & Challenge Question & $\begin{array}{c}\text { Rating } \\
(1-5)\end{array}$ \\
\hline 1. & $\begin{array}{l}\text { User question and answer entry (user defined) i.e } \\
\text { Question:1? answer: one }\end{array}$ & 5 \\
\hline 2. & One of your childhood friend's picture recognize name? & 5 \\
\hline 3. & How often do you go abroad? & 1 \\
\hline 4. & How many devices do you use for online transaction? & 5 \\
\hline 5. & Usually when do you use the online transaction? & 3 \\
\hline 6. & Your most transaction maximum limit? & 3 \\
\hline 7. & Your purpose of online transaction? & 1 \\
\hline 8. & Your highest educational degree? & 3 \\
\hline 9. & Which fruit do you like best? & 5 \\
\hline 10. & Which meal you enjoy more? & 5 \\
\hline 11. & $\begin{array}{l}\text { Your Passport No/Ration Card/ Aadhar Card/Driving } \\
\text { License? }\end{array}$ & 5 \\
\hline 12. & $\begin{array}{l}\text { Your Permanent Account } \\
\text { TIN/VAT ID Number? }\end{array}$ & 5 \\
\hline 13. & Your marriage date? & 5 \\
\hline 14. & What is your middle name? & 1 \\
\hline 15. & Your spouse was born in? & 3 \\
\hline 16. & Your first job in? & 1 \\
\hline 17. & Birthday of your spouse? & 3 \\
\hline 18. & The drink you like best? & 5 \\
\hline 19. & Are you going abroad in the coming month?(FAQ) & 5 \\
\hline 20. & Which color do you like best ? (FAQ) & 5 \\
\hline 21. & What is maximum Limit of your TP? & 5 \\
\hline 22. & Who is your favorite actor, musician or artist? & 3 \\
\hline 23. & What is your favorite place to visit as a child? & 3 \\
\hline 24. & What is your favorite song? & 5 \\
\hline 25. & What is the title of your favorite book? & 1 \\
\hline 26. & What was your most memorable gift as a child? & 5 \\
\hline 27. & What was the name of your first toy animal? & 3 \\
\hline 28. & What is your oldest sibling's middle name? & 1 \\
\hline 29. & What was the name of your elementary/ primary school? & 1 \\
\hline 30. & What is the country of your ultimate dream holiday? & 3 \\
\hline 31. & $\begin{array}{l}\text { What is the last name of your favorite secondary school } \\
\text { teacher? }\end{array}$ & 1 \\
\hline 32. & What was your dream job as a child? & 5 \\
\hline 33. & What is the name of your favorite childhood teacher? & 3 \\
\hline 34. & In which city or town was your first job? & 3 \\
\hline 35. & What was your secondary school mascot? & 5 \\
\hline 36. & Which month or day is your anniversary? & 5 \\
\hline
\end{tabular}

The outcome of the risk analysis decides the CQ selection as described in the Table II.

\section{TABLE II. CQ SELECTION PROCEDURE}

\begin{tabular}{|l|c|c|c|}
\hline \multicolumn{1}{|c|}{ Challenge Question } & $\begin{array}{c}\text { CQ } \\
\text { Level 1 }\end{array}$ & $\begin{array}{c}\text { CQ } \\
\text { Level 2 }\end{array}$ & $\begin{array}{c}\text { CQ } \\
\text { Level 3 }\end{array}$ \\
\hline Geo-location change & NO & NO & YES \\
\hline Transaction device change & NO & YES & NO \\
\hline Changing nature of transaction & YES & NO & NO \\
\hline Changing regular transaction purpose & YES & NO & NO \\
\hline Exceeding regular transaction frequency & NO & YES & NO \\
\hline Exceeding regular transaction limit & NO & NO & YES \\
\hline
\end{tabular}

\begin{tabular}{|c|c|c|c|}
\hline Challenge Question & $\begin{array}{c}C Q \\
\text { Level } 1\end{array}$ & $\begin{array}{c}C Q \\
\text { Level } 2\end{array}$ & $\begin{array}{c}\text { CQ } \\
\text { Level } 3\end{array}$ \\
\hline New user & YES & NO & $\mathrm{NO}$ \\
\hline Exceeding general transaction timing & NO & YES & YES \\
\hline First failure of login attempt & NO & NO & YES \\
\hline Second failure of login attempt & NO & YES & YES \\
\hline Third failure of login attempt & YES & YES & YES \\
\hline $\begin{array}{l}\text { Exceeding TP limit/ Forth failure of } \\
\text { login attempt }\end{array}$ & \multicolumn{3}{|c|}{$\begin{array}{c}\text { Temporarily Blocked and } \\
\text { notified }\end{array}$} \\
\hline
\end{tabular}

\section{Result AND Comparative ANAlysis}

This paper proposes an alternative method of authentication for financial services through the internet. It is a method to minimize financial fraud forgery on online financial network. The main challenge to avoid fraudulent activity in the financial network is to keep the system away from unauthorized person. The proposed method presented here includes sensitive personal information, which is called KYC information, to verify the actual owner of the account for online financial activity. It considers all the known and upcoming possible ways to theft information and unauthorized entry into the online financial system. The dimension to the risk of the hacking and information stealing is unlimited and tendency for these illegal operations is evolving from time to time; the method proposed is a way to extend the KYC database as required by risk assessment in a certain interval. As the FIs already preserved customers' KYC information, so it is effective and fruitful to continue the reuse of KYC data for authentication purpose rather than to bear additional cost involving mechanism.

By this approach, the evolving security threat can be minimized in a significant way. Moreover, the model performance is preserved and improved eventually in each user activity. Authentication measures are dynamically assigned to make the system more reliable and keep the unauthorized user out of the whole process. Thus it is mostly recommended to use Challenge Question (CQ) and One Time Password (OPT) described in Table III. CQ is ranked in various levels of standard ranging from 1 to 5 with an independent mechanism based on the heuristic result of the risk.

TABLE III. MEASURES FOR AUTHENTICATION

\begin{tabular}{|l|l|c|c|c|}
\hline \multicolumn{1}{|c|}{ Risk Group } & \multicolumn{1}{|c|}{ Risk Factor } & CQ & OTP & $\begin{array}{c}\text { E-mail } \\
\text { Confirmation }\end{array}$ \\
\hline Location Risk & Geo-location Change & $\checkmark$ & $\checkmark$ & $\checkmark$ \\
\hline Location Risk & $\begin{array}{l}\text { Transaction device } \\
\text { Change }\end{array}$ & $\checkmark$ & $\checkmark$ & \\
\hline Transaction Risk & $\begin{array}{l}\text { Exceeding Regular } \\
\text { transaction limit }\end{array}$ & $\checkmark$ & $\checkmark$ & $\checkmark$ \\
\hline Transaction Risk & $\begin{array}{l}\text { Exceeding Profile } \\
\text { transaction limit }\end{array}$ & Block Transaction and notify \\
\hline Behavioral Risk & $\begin{array}{l}\text { Regular transaction } \\
\text { timing transaction }\end{array}$ & $\checkmark$ & $\checkmark$ & $\checkmark$ \\
\hline Behavioral Risk & $\begin{array}{l}\text { Regular trancy } \\
\text { frequency transaction }\end{array}$ & $\checkmark$ & $\checkmark$ & \\
\hline Behavioral Risk & $\begin{array}{l}\text { Regular } \\
\text { purpose }\end{array}$ & $\checkmark$ & $\checkmark$ & \\
\hline Credential Risk & Nature of transaction & $\checkmark$ & $\checkmark$ & \\
\hline Credential Risk & $\begin{array}{l}\text { Changing KY } \\
\text { information }\end{array}$ & $\checkmark$ & $\checkmark$ & $\checkmark$ \\
\hline
\end{tabular}




\begin{tabular}{|c|l|c|c|c|}
\hline Risk Group & \multicolumn{1}{|c|}{ Risk Factor } & CQ & OTP & $\begin{array}{c}\text { E-mail } \\
\text { Confirmation }\end{array}$ \\
\hline Credential Risk & $\begin{array}{l}\text { User without details } \\
\text { identity (IP, Hardware } \\
\text { address, etc.) }\end{array}$ & \multicolumn{4}{|c|}{ Block Transaction and notify } \\
\hline
\end{tabular}

The CQ selection process after evaluating risk involved in the current activities of the user is explained using bar chart in Fig. 7. In the graph the CQ level 20 means the user is temporarily blocked. Other CQ level value will be assigned in the following way as KYC based CQ. i.e. if CQ Level $9=$ 1(CQ Level 1) + 3(CQ Level 3) + 5(CQ Level 5).

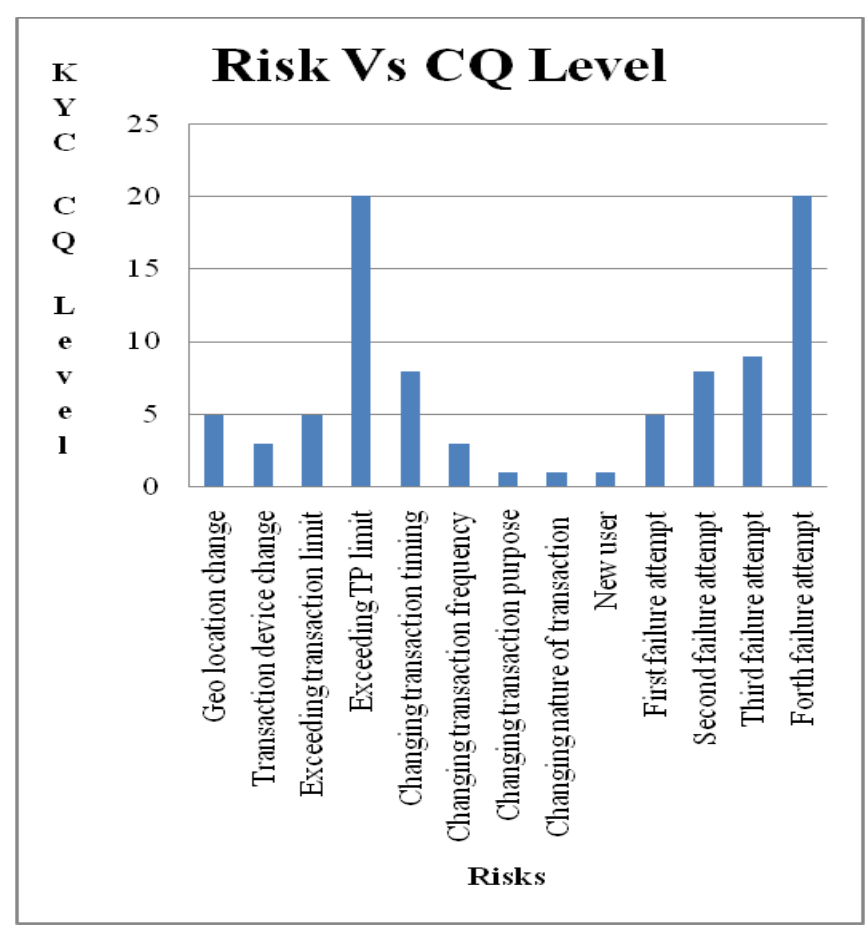

Fig. 7. CQ Level assignment based on the rise of the risk

In this paper user's own attributes are used to identify a real user and to recognize a verification method by using his/her own credentials from KYC information. KYC is the most secrete and private information to spot a particular account holder in the FIs and moreover, simulation shows mitigation of all possible ways of compromizing the system. It is well believed that the proposed system will ensure equal security as existing 2FA/MFA. Compare between the traditional scheme [12] and proposed model is shown in the TABLE IV.

TABLE IV. COMPARISON SECURITY PROPERTIES TRADITIONAL SCHEME AND THE PROPOSED MODEL

\begin{tabular}{|c|c|c|}
\hline Attacks & Traditional Scheme & CQ and/or OTP \\
\hline Evesdropping attack & & \\
\hline Replay attack & & \\
\hline Dictionary attack & & \\
\hline Brute force attack & & \\
\hline Man-in-the-middle attack & & \\
\hline User impersonation attack & & \\
\hline
\end{tabular}

KYC based CQ and OTP return a recommended model as it has multiple advantages with no chance to be stolen or lost, no chance to HASH change, and no chance to misguide by man-in-the-middle (MITM) attack.

\section{CONCLUSION}

In this paper, it has been proposed dynamic KYC based MFA authentication method to secure access of the financial services through the internet. Analysis and simulation results show that the proposed method provides control equal to existing MFA/2FA. The proposed method is costless and does not incur any hurdle to carry an additional hardware. As there is no chance of key theft, this method can be used on any private or public device. The method will ensure dynamic security by tackling most of the vulnerabilities in internet based financial transaction.

\section{REFERENCES}

[1] Wikipedia, "Know your customer (KYC) Laws by country".

[2] FATF, GAFI. "Guidance on the risk-based approach to combating money laundering and terrorist financing," June 2007.

[3] Federal Financial Institutions Examination Council (FFIEC), "Authentication in an Internet Banking Environment," Retrieved February 4, 2006.

[4] O.B. Lawal, A. Ibitola, O.B. Longe, "Internet banking authentication methods in Nigeria Commercial Banks," African Journal of Computing \& ICT,Vol 6. No. 1, March 2013.

[5] Shailesh Kumar Shivakumar, Babu Krishnamurthy, "Advanced security design for financial applications," External Document, 2016 [White Paper]

[6] R. Di Pietro, Gianluigi Me , M. A. Strangio, "A two-factor mobile authentication scheme for secure financial transactions," [International Conference on Mobile Business (ICMB'05), pp. 2834, 2005]

[7] Syeda Farha Shazmeen, Shyam Prasad, "A practical approach for secure internet banking based on cryptography," [International Journal of Scientific and Research Publications, Volume 2, Issue 12, December 2012]

[8] Paul Madsen, "Best practices for step-up multi-factor authentication," [White Paper]

[9] H. Alsaiari, M. Papadaki, P.S. Dowland , S.M. Furnell, "Alternative graphical authentication for online banking environments," [Proceedings of the Eighth International Symposium on Human Aspects of Information Security \& Assurance (HAISA 2014)]

[10] Lior Golan, Amir Orad, Naftali Bennett. "System and method for risk based authentication,” U.S. Patent US 20050097320 A1, May 5, 2005.

[11] Yaser Alosefer, Omer F. Rana, "Predicting client-side attacks via behaviour analysis using honeypot data," [Next Generation Web Services Practices (NWeSP), 2011 [7th International Conference on Next Generation Web Services Practices, pp.31,36, 19-21 Oct. 2011].

[12] Wen-Bin Hsieh, Jenq-Shiou Leu, "Design of a time and location based one-time password authentication scheme," 2011 [7th International Wireless Communications and Mobile Computing Conference (IWCMC) , 4-8 July 2011]

[13] World Bank dataset

Available:https://finances.worldbank.org/Financial-IntermediaryFunds/Financial-Intermediary-Funds-Cash-Transfers/h4s8-nwev

[14] Dataset from clients live website

Available: http://reporting4results.com/actiontracker/login.php 